Versorgungsstrukturgesetz

\title{
Viele Risiken und Nebenwirkungen
}

\section{Das Versorgungsstrukturgesetz (GKV-VSG) \\ ist für die Fachärzte kein „,großer Wurf". Bei einer Analyse der Pläne der schwarz-gel- ben Regierungskoalition zeigen sich aus der Sicht der Fachärzte viele Risiken und Nebenwirkungen.}

Der zentrale Punkt für die Fachärzte ist der Aufbau einer ambulanten spezialärztlichen Versorgung unabhängig und in Konkurrenz mit der allgemeinen fachärztlichen Versorgung. Hier werden die Fachärzte in einen knallharten Wettbewerb mit den Krankenhäusern gedrängt. Inzwischen gibt es massive Kritik der fachärztlichen Berufsverbände an diesem Vorhaben. Die systematische Trennung der fachärztlichen Versorgung in verschiedene Ebenen wird dabei als Spaltpilz für die Fachärzte gesehen. Das ambulante Leistungsspektrum der Fachärzte sei überwiegend sowohl auf sogenannte Versorgungsfälle als auch auf Spezialleistungen im Sinne der $\$ \$ 115 \mathrm{~b}$ und 116 SGB $\mathrm{V}$ ausgerichtet, somit einer krankenhausnahen Leistungserbringung, stellt die Gemeinschaft fachärztlicher Berufsverbände (GFB) fest.

Bei den Kassenärztlichen Vereinigungen formiert sich inzwischen der Widerstand gegen die Pläne des Bundesgesundheitsministers. Eine Resolution der Vertreterversammlung der Kassenärztlichen Vereinigung Niedersachsen findet breite Unterstützung. Gefordert werden hier folgende Nachbesserungen im Gesetzentwurf:

- Der Krankenhaus-Sektor muss an der Finanzierung zu gleichen Bedingungen beteiligt werden. Es darf keine Finanzierung der Vergütung spezialärztlicher Leistungen durch eine Bereinigung der Gesamtvergütung der Vertragsärzte geben.

- Eine Öffnung der Krankenhäuser für ambulante spezialärztliche Leistungen ist erst dann möglich, wenn eine einheitlich betriebswirtschaftlich kalkulierte Vergütung unter Berücksichtigung der Investitionskosten umgesetzt ist. Der geplante Investitionsabschlag von fünf Prozent ist für die Krankenhäuser völlig unzureichend. - Die Eingrenzung auf seltene Erkrankungen (fünf Erkrankungen pro eine Million Einwohner) und bestehende Versorgungsdefizite.

- Die Einzelleistungsvergütung zu festen Preisen ohne Mengenbegrenzung ist nötig.

- Die Abrechnung über die Kassenärztlichen Vereinigungen ist erforderlich.

- Die Beteiligung der Kassenärztlichen Vereinigungen bei der Festlegung der Qualitätsstandards und der Indikationsliste für die spezialärztliche Versorgung wird gefordert.

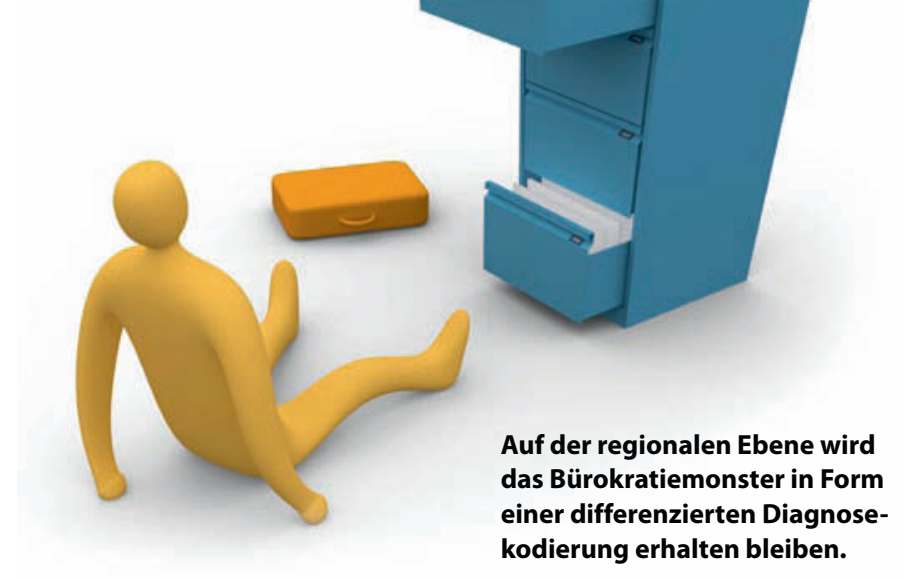

- Die Qualifikationsanforderungen aus dem ambulanten Bereich müssen auch für die Krankenhäuser mit voller Verbindlichkeit gelten. Der Facharzt-Standart ist gesetzlich festzuschreiben.

\section{Regionalisierung könnte Rückschlag sein}

Auch der Verzicht auf die Allgemeinen Kodierrichtlinien ist für die Fachärzte eine zweischneidige Sache. Bundesgesundheitsminister Daniel Bahr feiert diesen Bürokratieabbau als Erfolg für die Ärzte. Tatsächlich wird das „Bürokratiemonster“ aber nur auf der Bundesebene erfolgreich verjagt. Auf der regionalen Ebene werden die Ärzte sehr rasch wieder mit der Forderung der Krankenkassen konfrontiert, bei der Veränderung der morbiditätsbedingten Gesamtvergütung eine differenzierte Kodierung der Diagnosen vorzunehmen. Modellrechnungen lassen keinen Zweifel, dass die Fachärzte ein vitales Interesse daran haben müssen, dass die Veränderung der Morbidität bei den Zahlungen der Krankenkassen angemessen berücksichtigt wird.

Auch die Regionalisierung der Vertragsverhandlungen mit den Krankenkassen bedarf einer kritischen Bewertung. So manchen Facharzt treibt die Sorge um, dass die Verhandlungen der regionalen KV-Fürsten weniger erfolgreich sein werden, als die auf der Bundesebene unter der Verantwortung der Kassenärztlichen Bundesvereinigung. Die Regionalisierung könnte sich auch als Rückschlag für die Fachärzte in Regionen erweisen, die mit unterdurchschnittlichen Vergütungen konfrontiert sind. „Gleiches Geld für gleiche Leistungen“, diese Forderung wird auf Dauer ins Hintertreffen geraten, wenn die unterschiedliche Finanzkraft in den Bundesländern auf die Veränderung der Gesamtvergütungen und die Vergütung in den regionalen Euro-Gebührenordnungen ausstrahlt.

Norbert Fischer 